\title{
Comorbilità vera e comorbilità spuria nei disturbi dell'alimentazione
}

\author{
Riccardo Dalle Grave', Massimiliano Sartirana², Simona Calugi' \\ ' Department of Eating and Weight Disorders, Villa Garda Hospital \\ ${ }^{2}$ Associazione Disturbi Alimentari (ADA), Verona
}

\section{Parole chiave}

Disturbi dell'alimentazione Comorbilità

Terapia cognitivo comportamentale Depressione

Trattamento

\begin{abstract}
I ricercatori e i clinici spesso usano in modo acritico il termine "comorbilità" per descrivere i problemi clinici coesistenti con il disturbo dell'alimentazione, non valutando se sono "entità cliniche distinte" (comorbidità vera) o la diretta conseguenza del disturbo dell'alimentazione (comorbilità spuria) che scompare con la sua remissione. Inoltre, i clinici che lavorano nelle équipe multidisciplinari eclettiche non sempre valutano se i problemi clinici coesistenti ostacolino o meno il trattamento del disturbo dell'alimentazione perché non vedono l'intero quadro clinico del paziente e tendono a trattare solo le caratteristiche cliniche che sono di loro competenza. La valutazione e la gestione non accurata della comorbilità può avere l'effetto paradossale di defocalizzare il trattamento dai fattori chiave che mantengono la psicopatologia del disturbo dell'alimentazione e di sottoporre i pazienti a trattamenti inutili e potenzialmente dannosi.

In questo articolo sono affrontati quattro argomenti principali: (i) i dati disponibili sulla prevalenza della comorbilità nei disturbi dell'alimentazione; (ii) i problemi metodologici che affliggono la valutazione della comorbilità nei disturbi dell'alimentazione, che si riflettono sull'imbarazzante ampia variabilità dei risultati degli studi che l'hanno valutata; (iii) i rischi di una sovrastima della diagnosi di comorbilità quando i pazienti sono gestiti da terapeuti che non hanno una conoscenza approfondita della psicopatologia dei disturbi dell'alimentazione e delle sue conseguenze; (iv) l'approccio pragmatico della terapia cognitivo comportamentale migliorata (CBT-E) per la gestione della comorbilità nei disturbi dell'alimentazione.
\end{abstract}

\begin{abstract}
Researchers and clinicians often uncritically use the term "comorbidity" to describe the clinical problems coexisting with the eating disorder, not considering whether they are "distinct clinical entities" (i.e., true comorbidity) or the direct consequence of eating disorders (i.e., spurious co-morbidity) that disappear with its remission. Furthermore, clinicians working in multidisciplinary eclectic teams do not always assess whether coexisting clinical problems hinder or not the treatment of eating disorder because they do not see the entire clinical picture of the patient and tend to treat only the clinical features that are within their competence area. The inaccurate assessment and management of comorbidities may have the paradoxical effect to defocus the treatment from key factors that maintain the eating disorder psychopathology and to deliver to the patients unnecessary and potentially harmful treatments.

This article addresses four main topics: (i) the available data on the prevalence of comorbidity in eating disorders; (ii) the methodological problems affecting the assessment of comorbidity in eating disorders, which is reflected by the embarrassing wide variability of the results of the studies that evaluated it; (iii) the risks of overestimation of the diagnosis of co-morbidity when patients are managed by therapists who do not have a thorough knowledge of the psychopathology of eating disorders and its consequences; (iv) the pragmatic approach of enhanced cognitive behavioral therapy (CBT-E) in the management of comorbidity in eating disorders.
\end{abstract}

Copyright @ 2019 Riccardo Dalle Grave, Massimiliano Sartirana, Simona Calugi. This is an open-access article distributed under the terms of the Creative Commons Attribution License (CC BY). The use, distribution or reproduction in other forums is permitted, provided the original author(s) and the copyright owner(s) are credited and that the original publication in this journal is cited, in accordance with accepted academic practice. No use, distribution or reproduction is permitted which does not comply with these terms.

Riccardo Dalle Grave, MD (凶) rdalleg@gmail.com

Ricevuto: 10 Gennaio 2019; Accettato: 15 Gennaio 2019; Pubblicato online: 30 Gennaio 2019. doi: 10.32044/ijedo.2019.01 


\section{Introduzione}

Il termine comorbilità (o comorbidità) è stato coniato da Feinstein (1970) che lo ha definito "the existence or occurrence of any distinct additional entity during the clinical course of a patient who has the index disease under study". Questo termine è diventato di frequente uso e "di moda" in psichiatria per indicare non solo quei casi in cui un paziente riceve una diagnosi psichiatrica e una medica (per es. depressione maggiore e diabete di tipo 2), ma anche quei casi in cui il paziente riceve due o più diagnosi psichiatriche (per es. depressione maggiore e disturbo di panico) (Maj, 2005).

La comorbilità è un tema complesso, concettualmente e clinicamente. La definizione di comorbilità da un punto di vista concettuale si riferisce a una situazione in cui "un'entità clinica distinta si sviluppa durante il decorso di una malattia" (per es. quando un paziente con diabete mellito sviluppa la malattia di Parkinson). In questo caso sono presenti due entità cliniche distinte e si fa riferimento a un concetto lifetime. La definizione di comorbilità da un punto di vista clinico si riferisce, invece, a una situazione in cui "coesistono due o più entità cliniche distinte". In questo caso la prevalenza della comorbilità dipende dalla definizione dei disturbi (cioè il sistema classificativo e le sue regole diagnostiche). Nel campo delle malattie mentali, in cui non sono stati fino ad ora trovati bio-marcatori specifici, è discutibile se esse siano entità cliniche "distinte" (Maj, 2005) oppure siano semplicemente il risultato dell' attuale classificazione che, sulla base dei criteri diagnostici descritti nel Manuale Statistico dei Disturbi Mentali (DSM) (American Psychiatric Association, 2013), spinge, in base ai sintomi presentati, ad applicare più diagnosi psichiatriche nello stesso paziente, quasi a sottolineare la nostra attuale tendenza a formulare, forse inutilmente, più ipotesi di quelle che siano necessarie per spiegare un dato fenomeno quando quelle iniziali sono sufficienti, argomentazione alla base del principio metodologico del Rasoio di Occam (Murray, Loeb, \& Le Grange, 2018).

I problemi legati alla definizione di comorbilità possono avere importanti conseguenze cliniche che si ripercuotono sul trattamento. Per esempio, le caratteristiche della depressione sono comuni nei pazienti con disturbi dell'alimentazione, ma possono essere l'evidenza di una depressione clinica coesistente (comobildità vera) oppure la diretta conseguenza del disturbo dell'alimentazione (comorbilità spuria). Nel primo caso, la depressione clinica va trattata direttamente, mentre nel secondo il trattamento del disturbo dell'alimentazione dovrebbe determinare la remissione delle caratteristiche depressive.

\section{Comorbilità nei disturbi dell'alimentazione: dati epidemiologici}

Una revisione narrativa ha recentemente riassunto gli studi europei che hanno valutato la prevalenza della comorbilità psichiatrica nei disturbi dell'alimentazione (Keski-Rahkonen \& Mustelin, 2016). La comorbilità è stata diagnosticata in oltre il $70 \%$ delle persone con disturbo dell'alimentazione e i disturbi più frequenti coesistenti sono i seguenti: disturbi d'ansia $(>50 \%)$, disturbi dell'umore $(>40 \%)$, autolesionismo (>20\%) e disturbo da uso di sostanze (>10\%). Molto frequente sembra essere la presenza lifetime di ossessioni e compulsioni che, in uno studio eseguito su pazienti con anoressia nervosa, è stata evidenziata in circa il $70 \%$ dei casi (Halmi et al., 2003). È stata anche trovata un'aggregazione tra disturbi dello spettro autistico nei probandi con anoressia nervosa e i loro parenti (Koch et al., 2015). Infine, c'è qualche evidenza dell'esistenza di un'associazione tra disturbo da deficit di attenzione e iperattività e disturbi dell'alimentazione (Sala et al., 2018). Va sottolineato che gli studi effettuati presentano un'ampia variabilità nel tasso di comorbilità psichiatrica nei disturbi dell'alimentazione; per esempio, la prevalenza di una storia lifetime di un disturbo d'ansia è stata riportata nel $25-75 \%$ dei casi (Swinbourne \& Touyz, 2007): un dato che getta importanti dubbi sull'attendibilità di queste osservazioni.

Gli studi cha hanno riportato la prevalenza dei disturbi di personalità coesistenti con i disturbi dell'alimentazione hanno riportato una variabilità ancora più ampia che va dal 27\% al 93\% (Vitousek \& Manke, 1994). L'anoressia nervosa è stata più frequentemente associata al disturbo evitante di personalità - Cluster $\mathrm{C}$ ansioso/pauroso, mentre la bulimia nervosa è più frequentemente associata ai disturbi di personalità del Cluster B - drammatico/erratico/emotivo. Una recente metanalisi, che ha trovato una prevalenza del $53 \%$ di disturbi di personalità nei pazienti con qualsiasi tipo di disturbo dell'alimentazione rispetto al $9 \%$ nei controlli sani, non ha però trovato differenze statisticamente significative nella prevalenza totale di disturbi di personalità tra anoressia nervosa (49\%) e bulimia nervosa (54\%) e nei vari cluster di disturbi di personalità, eccetto per il disturbo di personalità ossessivo-compulsivo che è risultato essere più prevalente nell'anoressia nervosa (23\%) rispetto alla bulimia nervosa (12\%) (Martinussen et al., 2017). 


\section{Comorbilità nei disturbi dell'alimentazione: problemi metodologici}

Gli studi che hanno valutato la comorbilità nei disturbi dell'alimentazione soffrono di gravi problemi metodologici che si riflettono sull'imbarazzante ampia variabilità dei risultati che hanno valutato questo problema. In sintesi, $\mathrm{i}$ principali difetti metodologici sono i seguenti:

- Insorgenza cronologica: non sempre è stata fatta una distinzione se il disturbo in comorbilità è insorto prima o dopo il disturbo dell'alimentazione.

- Campioni piccoli: i campioni valutati sono, nella maggior parte dei casi, di piccole dimensioni e con basso potere statistico per trarre delle conclusioni attendibili.

- Campioni clinici: nella maggior parte degli studi sono stati valutati campioni clinici in cui la prevalenza di comorbilità psichiatrica è verosimilmente maggiore rispetto ai campioni non clinici.

- Composizione dei campioni: i campioni valutati comprendevano le categorie diagnostiche dei disturbi dell'alimentazione in proporzioni diverse.

- Strumenti diversi: sono stati usati strumenti diversi per valutare la comorbilità (un ampio ed eterogeneo numero di interviste diagnostiche e di test autosomministrati).

- Limitato uso di gruppi di controllo: raramente è stato incluso negli studi un gruppo di controllo pareggiato per sesso ed età.

- Assenza di valutazione degli effetti secondari del disturbo dell'alimentazione: quasi mai è stato valutato se le caratteristiche di comorbilità fossero secondarie al disturbo dell'alimentazione.

- Diagnosi eseguita dai ricercatori o dai clinici: la prevalenza della comorbilità può variare se la diagnosi è effettuata da ricercatori che usano strumenti standardizzati o da clinici che non li usano.

Nei disturbi di personalità, inoltre, numerosi problemi complicano l'interpretazione dei dati sulla personalità nei pazienti con disturbi dell'alimentazione (Vitousek \& Manke, 1994). In particolare, è difficile valutare la vera personalità dei pazienti con disturbi dell'alimentazione perché è influenzata dalla psicopatologia specifica e dalle conseguenze della malnutrizione (Garner \& Dalle Grave, 1999). Inoltre, è azzardato fare una diagnosi di disturbi di personalità coesistente perché i pazienti con disturbi dell'alimentazione, che hanno un esordio del loro disturbo dell'alimentazione in giovane età, non hanno periodi liberi dalla presenza del disturbo dell'alimentazione (Fairburn, 2008).

\section{Comorbilità o casi complessi?}

L'uso del termine "comorbilità", per indicare la concomitanza di due o più diagnosi psichiatriche, è considerato sbagliato da alcuni autori perché nella maggior parte dei casi non è chiaro se le diagnosi concomitanti riflettano la presenza di entità cliniche distinte $\mathrm{o}$ facciano riferimento $\mathrm{a}$ manifestazioni multiple di una singola entità clinica (Maj, 2005). È un dato di fatto che il riscontro di più diagnosi psichiatriche coesistenti è diventato via via più frequente. Questo è in parte la conseguenza dell'uso di interviste diagnostiche standardizzate, che aiuta a identificare diversi aspetti clinici, che in passato erano rimasti inosservati dopo la diagnosi principale, ma verosimilmente è la conseguenza dell'attuale sistema classificativo DSM-5 per almeno quattro motivi principali (Maj, 2005):

1. L'uso della regola implicita che lo stesso sintomo non deve apparire in più di un disturbo (per es., il sintomo d'ansia non deve apparire nei criteri diagnostici della depressione maggiore, sebbene le persone affette da depressione abbiano spesso ansia).

2. La proliferazione di nuove categorie diagnostiche psichiatriche.

3. L'uso limitato di categorie diagnostiche gerarchiche.

4. Basare la diagnosi su criteri operativi piuttosto che su criteri basati su descrizioni cliniche.

La divisone artificiosa in piccoli pezzi di condizioni cliniche complesse ha l'effetto negativo di prevenire un approccio più olistico alla persona e di favorire un uso ingiustificato di più farmaci per trattare singoli pezzi di un quadro clinico più ampio e complesso (Maj, 2005). Per questo motivo, per i disturbi mentali sembra sia più opportuno e clinicamente utile parlare di "casi complessi" piuttosto che di comorbilità.

La nozione che esista solo un sottogruppo di "casi complessi” non si può applicare ai disturbi dell'alimentazione (Fairburn, 2008). Infatti, quasi tutti i pazienti affetti da disturbi dell'alimentazione possono essere considerati dei casi complessi. La maggior parte, come descritto sopra, soddisfa i criteri diagnostici per uno o più disturbi psichiatrici DSM-5, in particolare per la depressione clinica, i disturbi d'ansia e i disturbi da uso di sostanze, e molti attraggono la diagnosi di qualche disturbo di personalità. Infine, le complicazioni fisiche sono comuni e un sottogruppo ha una co-esistente e interagente patologia medica. Le difficoltà interpersonali sono la norma e l'andamento cronico del disturbo può avere un impatto fortemente ne- 
gativo sullo sviluppo e sul funzionamento interpersonale della persona. Tutto questo evidenzia che nei pazienti affetti da disturbi dell'alimentazione la complessità è la norma piuttosto che l'eccezione (Fairburn, 2008).

\section{La gestione acritica della comorbilità da parte delle équipe multidisciplinari eclettiche}

La valutazione e gestione dei disturbi psichiatrici e internistici coesistenti ai disturbi dell'alimentazione necessita, spesso, il coinvolgimento di più professionisti perché sono richieste molteplici e complesse competenze professionali e inevitabilmente un approccio multidisciplinare. Tuttavia, la maggior parte delle équipe multidisciplinari adotta un approccio "eclettico" che può includere una vasta gamma di procedure mediche, psichiatriche, psicologiche ed educative derivanti spesso da teorie diverse, a volte contrastanti tra loro. In questo contesto, per esempio, lo psicologo può somministrare una psicoterapia psicoanalitica, il dietista utilizzare procedure di tipo comportamentale, lo psichiatra valutare e curare la comorbilità con psicofarmaci e il medico internista gestire le complicanze e la comorbilità internistica. In altre parole, ogni membro dell'équipe segue la teoria e la pratica che ha appreso nel proprio percorso di studi, perseguendo obiettivi terapeutici relativi al proprio ruolo professionale piuttosto che quelli dell'équipe nel suo complesso.

Mentre ciascuno di questi singoli approcci può avere qualche merito e, in una certa misura, fornire qualche beneficio ai pazienti, i trattamenti multidisciplinari "eclettici" privi di un modello teorico di riferimento spesso affrontano in modo acritico i problemi clinici psichiatrici e internistici coesistenti, ponendo scarsa attenzione al fatto se essi rappresentino una comorbilità reale o una comorbilità spuria determinata dagli effetti della psicopatologia dei disturbi dell'alimentazione e/o della malnutrizione (Calugi, Chignola, El Ghoch, \& Dalle Grave, 2018). Inoltre, nei trattamenti multidisciplinari eclettici è raramente valutato se i problemi clinici coesistenti ostacolino o meno il trattamento del disturbo dell'alimentazione perché i vari specialisti coinvolti non vedono l'intero quadro clinico della paziente e trattano solo le caratteristiche cliniche presenti che sono di loro competenza. La diagnosi di comorbilità è ancora più frequente se la sua valutazione è eseguita da uno psichiatra o un internista generalista che non conosce la psicopatologia dei disturbi dell'alimentazione e tende ad attribuire alla comorbilità le conseguenze del disturbo dell'alimentazione.
Una gestione acritica della comorbilità può avere, tra le altre cose, l'effetto di defocalizzare il trattamento dai fattori chiave che mantengono la psicopatologia del disturbo dell'alimentazione e di sottoporre la paziente a trattamenti inutili e potenzialmente dannosi. Nello specifico gli errori terapeutici che più frequentemente abbiamo osservato nella nostra pratica clinica sono il trattamento con una varietà di farmaci, senza per altro ottenere dei benefici, delle conseguenze fisiche e psicosociali della malnutrizione, per esempio somministrando estroprogestinici per la cura dell'amenorrea secondaria, ansiolitici, antidepressivi, neurolettici e stabilizzatori del tono dell'umore per trattare l'ansia, l'irritabilità, la deflessione del tono dell'umore e l'insonnia, sintomi che nella maggior parte dei casi sono la conseguenza della malnutrizione e/o della psicopatologia del disturbo dell'alimentazione. Altri tipi di errori sono spesso effettuati con alcuni trattamenti psicologici che affrontano soltanto le conseguenze psicosociali del disturbo, per esempio le difficoltà relazionali o emotive, ma non direttamente la psicopatologia specifica disturbo dell'alimentazione.

\section{L'approccio pragmatico della CBT-E nella gestione dei casi complessi}

Per far fronte ai problemi sopra descritti, la versione italiana della terapia cognitivo comportamentale (CBT-E), applicata a veri livelli di cura (ambulatorio, day-hospital e ricovero) e chiamata CBT-E multistep, è stata progettata per garantire un approccio multidisciplinare "non eclettico" in cui tutti i terapeuti coinvolti (per es. medici, psichiatri, psicologi, dietisti e infermieri) hanno ricevuto una formazione completa nella CBT-E prima di unirsi all'équipe (Dalle Grave, 2015). Questo permette ai terapeuti di continuare a mantenere il loro specifico ruolo professionale, ma di condividere la stessa cornice teorica (la teoria transdiagnostica e la CBT-E) e di utilizzare un linguaggio comune con i pazienti. Così, dopo le sedute di valutazione e preparazione in cui un medico esperto dell'équipe è coinvolto per valutare se l'eventuale comorbilità associata al disturbo dell'alimentazione sia reale o spuria, la CBT-E può essere somministrata sia da un singolo terapeuta (CBT-E ambulatoriale) che da un'équipe multidisciplinare non eclettica (CBT-E ambulatoriale intensiva e CBT-E ospedaliera). Inoltre, sebbene la maggior parte dei casi ambulatoriali richieda l'intervento di un singolo terapeuta, in questo modello di cura le periodiche valutazioni eseguite da un medico dell'équipe possono essere integrate nel trattamento senza interrompere il processo terapeutico. Allo 
stesso modo, gli interventi del medico per trattare i disturbi psichiatrici e medici coesistenti (per es. depressione clinica, obesità, diabete ecc.) o le complicazioni associate al basso peso e/o all'uso dei comportamenti eliminativi, sono integrati coerentemente nella CBT-E e concordati sempre con il terapeuta che la somministra. La Tabella 1 mostra le linee guida pragmatiche, a cui tutti i terapeuti dell'équipe si rifanno, che si basano sul principio generale secondo cui la comorbilità va riconosciuta ed eventualmente trattata solo quando è significativa e ha implicazioni cliniche.

\section{II caso speciale della depressione clinica}

La coesistenza dei disturbi dell'alimentazione con i disturbi dell'umore è stata riportata in più del $40 \%$ dei casi (Keski-Rahkonen \& Mustelin, 2016), ma spesso la diagnosi di depressione clinica coesistente è impropria (Fairburn et al., 2008). Questo accade perché c'è una sostanziale sovrapposizione concettuale tra i due disturbi e non è semplice capire se la coesistenza di un disturbo dell'alimentazione e della depressione clinica costituisca una comorbilità vera o spuria.
Nell'anoressia nervosa molte caratteristiche cliniche rilevate sono la conseguenza della malnutrizione e del sottopeso (Keys et al., 1950). Esempi sono: umore depresso, isolamento sociale, aumento dell'ossessività e dell'indecisione, sonno disturbato con risvegli precoci, mancanza di energia e perdita del desiderio sessuale. Nella bulimia nervosa molte caratteristiche cliniche evidenziate sono, invece, la conseguenza degli episodi ricorrenti di abbuffata (Fairburn et al., 2008). Esempi sono: autocritica, umore depresso, isolamento sociale e sensazione di impotenza. In entrambi i casi, queste manifestazioni cliniche possono essere facilmente confuse con una vera e propria depressione clinica, quando in realtà, sono effetti secondari del disturbo dell'alimentazione.

Al contrario, caratteristiche suggestive che fanno sospettare la presenza di depressione clinica coesistente al disturbo dell'alimentazione sono le seguenti (Fairburn et al., 2008):

- Presenza di una storia personale di depressione clinica che precede il disturbo dell'alimentazione

- Insorgenza tardiva del disturbo dell'alimentazione

- Recente e non spiegata intensificazione delle caratteristiche depressive in assenza di modificazioni della

Tabella 1. Linee guida pragmatiche adottate dalla CBT-E per la gestione della comorbilità medica e psichiatrica associata al disturbo dell'alimentazione

Quando si osservano le caratteristiche che potrebbero far pensare alla presenza di un disturbo psichiatrico coesistente il clinico dovrebbe fare le seguenti valutazioni:

1. Le caratteristiche del disturbo coesistente sono attribuibili al disturbo dell'alimentazione o alle sue conseguenze e non interferiscono con il trattamento. Se è così, la comorbilità apparente potrebbe essere spuria e rappresentare semplicemente la caratteristica del disturbo dell'alimentazione. Questi disturbi vanno riconosciuti, monitorati e rivalutati durante il trattamento, ma ad essi non viene data un'attenzione speciale. Esempi sono:

a. Depressione clinica secondaria (attribuibile al disturbo dell'alimentazione)

b. Ansia sociale (attribuibile al disturbo dell'alimentazione)

c. Malnutrizione

d. Diabete mellito instabile

2. Le caratteristiche del disturbo coesistente non interferiscono probabilmente con il trattamento del disturbo dell'alimentazione, ma non rispondono ad esso. In questo caso, i disturbi vanno riconosciuti, e viene presa una decisione su quando affrontarli. Come regola generale è consigliabile affrontarli prima o dopo la psicoterapia del disturbo dell'alimentazione, soprattutto se sono trattati con una psicoterapia. Esempi sono:

a. Disturbo post-traumatico da stress, incluso abuso sessuale riferito

b. Disturbo ossessivo compulsivo (in questo caso può essere associato alla psicoterapia del disturbo dell'alimentazione un SSRI)

c. Obesità

3. Le caratteristiche del disturbo coesistente interferiscono probabilmente con il trattamento. Questi disturbi devono essere riconosciuti e affrontati prima di iniziare il trattamento. Esempi sono:

a. Uso di sostanze continuativo.

b. Disturbi psicotici acuti.

c. Depressione clinica (non attribuibile al disturbo dell'alimentazione). 
psicopatologia del disturbo dell'alimentazione (per es. accentuazione dell'umore depresso, isolamento sociale, ideazione suicidaria)

- Presenza di perdita di stimoli, pianto, ricorrenti pensieri sull'inutilità della vita, trascuratezza personale.

La distinzione tra depressione secondaria al disturbo dell'alimentazione e depressione clinica coesistente è essenziale per l'intervento terapeutico. Nel primo caso, non dovrebbe essere trattata perché la sua risoluzione è conseguente al miglioramento della psicopatologia del disturbo dell'alimentazione. Il rischio nel cercare di trattare questi effetti secondari con un altro intervento terapeutico (farmacologico o psicologico) è quello di distogliere il paziente e il terapeuta dal lavoro sul disturbo dell'alimentazione e, comunque, di non ottenere miglioramenti significativi.

$\mathrm{Al}$ contrario, la depressione clinica coesistente va identificata e trattata perché ostacola il trattamento del disturbo dell'alimentazione. Chi soffre di depressione clinica coesistente, infatti, pensa che non sia possibile cambiare, ha poca energia per impegnarsi nel trattamento e ha scarsa concentrazione per comprendere e trattenere le informazioni.

Nei casi di depressione clinica coesistente al disturbo dell'alimentazione viene raccomandato, dopo aver educato la paziente e raccolto il suo consenso, un trattamento farmacologico con antidepressivi da mantenere per almeno 9-12 mesi. La scelta di usare i farmaci e non una psicoterapia per la cura della depressione clinica dipende da due osservazioni (Fairburn et al., 2008): (i) il trattamento psicologico della depressione clinica richiede molto tempo e il progresso è limitato dal disturbo dell'alimentazione (si crea un'interazione negativa tra i due disturbi) e (ii) i farmaci antidepressivi, in particolare gli SSRI (per es. sertralina e fluoxetina) funzionano generalmente bene e rapidamente.

Se è presente una depressione clinica coesistente e la paziente ha riserve nell'usare gli antidepressivi va informata che essi non interferiscono con la capacità di fare le cose della vita e che la risoluzione della depressione clinica permetterà di beneficiare a pieno di una psicoterapia per il disturbo dell'alimentazione. Inoltre, gli antidepressivi non creano dipendenza, non aumentano il tono dell'umore (trattano solo la depressione) e hanno pochi effetti collaterali, come la nausea (effetto collaterale transitorio che dura qualche giorno). Se altri effetti collaterali, come il tremore fine alle mani, la difficoltà a deglutire e la diminuzione o la perdita del desiderio sessuale, sono persistenti, va valutata la possibilità di cambiare l'antidepressivo. Gli antidepressivi SSRI non aumentano l'appetito, c'è solo una maggiore sensibilità agli effetti inebrianti dell'alcool per cui bisogna bere con cautela.

Nel caso in cui la paziente rifiuti l'assunzione di antidepressivi può essere proposto un trattamento residenziale basato sulla CBT-E. Uno studio da noi eseguito ha, infatti, evidenziato che non ci sono differenze nell'esito a breve e a lungo termine nelle pazienti affette da disturbi dell'alimentazione con o senza depressione clinica (Calugi, El Ghoch, Conti, \& Dalle Grave, 2014).

\section{Conclusioni}

I disturbi dell'alimentazione sono per la loro natura quasi sempre "casi complessi" perché nella maggior parte dei pazienti sono presenti altri problemi medici e psichiatrici coesistenti. La comorbilità psichiatrica, in particolare, è stata riportata in oltre il $70 \%$ delle persone con disturbo dell'alimentazione (Keski-Rahkonen \& Mustelin, 2016). La valutazione della comorbilità è però afflitta da numerosi problemi metodologici che si riflettono sull'imbarazzante ampia variabilità dei risultati che hanno valutato questo problema. Nei clinici, in particolare in quelli che non hanno una conoscenza approfondita della psicopatologia specifica dei disturbi dell'alimentazione e delle sue conseguenze o che operano in équipe multidisciplinare eclettiche, l'errore più comune è il trattare come comorbilità vera alcune caratteristiche che sono la mera conseguenza del disturbo dell'alimentazione (comorbilità spuria), che scompaiono con la sua remissione. L'approccio acritico nella gestione della comorbilità ha delle potenziali conseguenze negative che possono influenzare negativamente l'esito del trattamento perché espongono i pazienti a trattamenti inutili e a volte dannosi che hanno spesso l'effetto di defocalizzare il paziente dall'affrontare i meccanismi di mantenimento della psicopatologia del disturbo dell'alimentazione.

Per far fronte a questi problemi la CBT-E ha adottato un approccio che include la somministrazione del trattamento da parte di équipe multidisciplinari non eclettiche e l'adozione di linee guida pragmatiche che indicano se e quando trattare la comorbilità. In questo modo l'intervento del medico per trattare i disturbi psichiatrici e medici coesistenti (o le complicazioni associate al basso peso e/o all'uso dei comportamenti eliminativi) sono integrati coerentemente nella CBT-E e concordati sempre con il terapeuta che la somministra. 


\section{Referenze}

American Psychiatric Association. (2013). Diagnostic and statistical manual of mental disorders, (DSM-5). Arlington: American Psychiatric Publishing.

Calugi, S., Chignola, E., El Ghoch, M., \& Dalle Grave, R. (2018). Starvation symptoms in patients with anorexia nervosa: a longitudinal study. Eat Disord, 1-15. doi:10. 1080/10640266.2018.1471921.

Calugi, S., El Ghoch, M., Conti, M., \& Dalle Grave, R. (2014). Depression and treatment outcome in anorexia nervosa. Psychiatry Research, 218(1-2), 195-200. doi:10.1016/j.psychres.2014.04.024.

Dalle Grave, R. (2015). La terapia cognitivo comportamentale multistep dei disturbi dell'alimentazione. Teoria, trattamento e casi clinici. Firenze: Eclipsi.

Fairburn, C. G. (2008). Cognitive behavior therapy and eating disorders. New York: Guilford Press.

Fairburn, C. G., Cooper, Z., \& Waller, D. (2008). Complex cases and comorbidity. In C. G. Fairburn (Ed.), Cognitive behavior therapy and eating disorders. New York: Guilford Press.

Feinstein, A. R. (1970). The pre-therapeutic classification of co-morbidity in chronic disease. Journal of Chronic Diseases, 23(7), 455-468.

Garner, D. M., \& Dalle Grave, R. (1999). Terapia cognitivo comportamentale dei disturbi dell'alimentazione. Verona: Positive Press.

Halmi, K. A., Sunday, S. R., Klump, K. L., Strober, M., Leckman, J. F., Fichter, M., ... Kaye, W. H. (2003). Obsessions and compulsions in anorexia nervosa subtypes. International Journal of Eating Disorders, 33(3), 308-319. doi:10.1002/eat.10138.

Keski-Rahkonen, A., \& Mustelin, L. (2016). Epidemiology of eating disorders in Europe: prevalence, incidence, comorbidity, course, consequences, and risk factors. Curr Opin Psychiatry, 29(6), 340-345. doi:10.1097/ yco.0000000000000278.
Keys, A., Brozek, J., Henschel, A., Mickelsen, O., \& Taylor, H. (1950). The Biology of Human Starvation. Minneapolis: University of Minnesota Press.

Koch, S. V., Larsen, J. T., Mouridsen, S. E., Bentz, M., Petersen, L., Bulik, C., ... Plessen, K. J. (2015). Autism spectrum disorder in individuals with anorexia nervosa and in their first- and second-degree relatives: Danish nationwide register-based cohort-study. British Journal of Psychiatry, 206(5), 401-407. doi:10.1192/bjp. bp.114.153221.

Maj, M. (2005). "Psychiatric comorbidity": an artefact of current diagnostic systems? British Journal of Psychiatry, 186, 182-184. doi:10.1192/bjp.186.3.182.

Martinussen, M., Friborg, O., Schmierer, P., Kaiser, S., Overgard, K. T., Neunhoeffer, A. L., ... Rosenvinge, J. H. (2017). The comorbidity of personality disorders in eating disorders: a meta-analysis. Eating and Weight Disorders, 22(2), 201-209. doi:10.1007/s40519-0160345-x.

Murray, S. B., Loeb, K. L., \& Le Grange, D. (2018). Treatment outcome reporting in anorexia nervosa: time for a paradigm shift? Journal of Eating Disorders, 6(1), 10. doi:10.1186/s40337-018-0195-1.

Sala, L., Martinotti, G., Carenti, M. L., Romo, L., Oumaya, M., Pham-Scottez, A., ... Janiri, L. (2018). Attention-deficit/hyperactivity disorder symptoms and psychological comorbidity in eating disorder patients. Eating and Weight Disorders, 23(4), 513-519. doi:10.1007/s40519-017-0395-8.

Swinbourne, J. M., \& Touyz, S. W. (2007). The co-morbidity of eating disorders and anxiety disorders: a review. Eur Eat Disord Rev, 15(4), 253-274. doi:10.1002/ erv.784.

Vitousek, K., \& Manke, F. (1994). Personality variables and disorders in anorexia nervosa and bulimia nervosa. Journal of Abnormal Psychology, 103(1), 137-147. 